\title{
Efficient genome editing of genes involved in neural crest development using the CRISPR/Cas9 system in Xenopus embryos
}

\author{
Zhongzhen Liu ${ }^{1,5}$, Tina Tsz Kwan Cheng ${ }^{1}$, Zhaoying Shi' ${ }^{2}$, Ziran Liu' ${ }^{1}$, Yong Lei ${ }^{1}$, Chengdong Wang ${ }^{1}$, Weili Shi ${ }^{1}$,
} Xiongfeng Chen ${ }^{3}$, Xufeng Qi, ${ }^{4,6}$, Dongqing Cai ${ }^{4,6}$, Bo Feng ${ }^{1}$, Yi Deng ${ }^{2}$, Yonglong Chen ${ }^{2^{*}}$ and Hui Zhao ${ }^{1,5^{*}}$

\begin{abstract}
Background: The RNA guided CRISPR/Cas9 nucleases have been proven to be effective for gene disruption in various animal models including Xenopus tropicalis. The neural crest (NC) is a transient cell population during embryonic development and contributes to a large variety of tissues. Currently, loss-of-function studies on NC development in $X$. tropicalis are largely based on morpholino antisense oligonucleotide. It is worthwhile establishing targeted gene knockout $X$. tropicails line using CRISPR/Cas9 system to study NC development.
\end{abstract}

Methods: We utilized CRISPR/Cas9 to disrupt genes that are involved in NC formation in X. tropicalis embryos. A single sgRNA and Cas 9 mRNA synthesized in vitro, were co-injected into $X$. tropicalis embryos at one-cell stage to induce single gene disruption. We also induced duplex mutations, large segmental deletions and inversions in $X$. tropicalis by injecting Cas9 and a pair of sgRNAs. The specificity of CRISPR/Cas9 was assessed in X. tropicalis embryos and the Cas9 nickase was used to reduce the off-target cleavages. Finally, we crossed the $\mathrm{G} 0$ mosaic frogs with targeted mutations to wild type frogs and obtained the germline transmission.

Results: Total 16 target sites in 15 genes were targeted by CRISPR/Cas 9 and resulted in successful indel mutations at 14 loci with disruption efficiencies in a range from 9.3 to $57.8 \%$. Furthermore, we demonstrated the feasibility of generation of duplex mutations, large segmental deletions and inversions by using Cas 9 and a pair of sgRNAs. We observed that CRISPR/Cas9 displays obvious off-target effects at some loci in X. tropicalis embryos. Such off-target cleavages was reduced by using the D10A Cas9 nickase. Finally, the Cas9 induced indel mutations were efficiently passed to G1 offspring.

Conclusion: Our study proved that CRISPR/Cas9 could mediate targeted gene mutation in X. tropicalis with high efficiency. This study expands the application of CRISPR/Cas9 platform in X. tropicalis and set a basis for studying NC development using genetic approach.

Keywords: Genome editing, Cas9, Neural crest, Gene disruption, Segmental deletion/inversion, Multiplex deletion, Xenopus

\footnotetext{
*Correspondence: chenyl@sustc.edu.cn; zhaohui@cuhk.edu.hk

${ }^{1}$ Key Laboratory for Regenerative Medicine, Ministry of Education, School

of Biomedical Sciences, Faculty of Medicine, The Chinese University

of Hong Kong, Hong Kong, SAR, China

${ }^{2}$ Shenzhen Key Laboratory of Cell Microenvironment, Department

of Biology, South University of Science and Technology of China,

Shenzhen, Guangdong 518055, China

Full list of author information is available at the end of the article
} 


\section{Background}

The neural crest (NC) is a unique cell population that are pluripotency and highly migratory, and can differentiate into a large variety of cell types including the peripheral nervous system and facial skeletons. During embryonic development, the NC specification, migration and differentiation are tightly regulated by a sophisticated regulatory network [1]. The NC development has been intensively studied using various animal models including the Хenopus embryos, however, currently loss-offunction assay for studying NC development in Xenopus embryos are largely based on the knockdown approach of morpholino antisense oligonucleotides. Therefore, generation of gene knockout genetic model of Xenopus will provide new insights on NC development.

Precise and efficient genome editing by engineered endonucleases is considered as a powerful reverse genetic approach to facilitate the study of gene functions and to establish disease models. The currently available genome editing tools of engineered endonucleases like Zinc Finger Nucleases (ZFNs) and Transcription Activator-Like Effector Nucleases (TALENs) have enabled relatively fast, specific genome targeting across human cells and various model organisms [2-9]. In addition, the newly emerged platform based on the type II clustered regularly interspaced short palindromic repeats (CRISPR) system offers a RNA-guided DNA recognition platform over the current protein based platforms of ZFNs and TALENs for genetic manipulation because of its convenience and easy access features [10-13]. This bacterial based adaptive immune system consists of two short RNAs, tracrRNA and crRNA, and a single protein Cas9. The tracrRNA and crRNA can be fused as one single guide RNA (sgRNA) for a functional CRISPR system in a previous study [12]. The commonly used Cas9 is derived from Streptococcus pyogenes (hereafter, Cas9 refers to the spCas9). The target sequence of Cas9 can be any 20 bp sequence followed by NGG, a conserved protospacer adjacent motif (PAM) [11, 13], which restricts the S. pyogenes Cas9 target space to every eight base pairs on average. By now, the CRISPR systems have been shown to disrupt specific targets effectively in various cell lines and organisms [11, 14-26]. Co-injection of more than one custom sgRNAs with Cas 9 mRNA demonstrated multiplexed gene disruption in mouse embryonic stem cells [27], zebrafish $[27,28]$ and X. tropicalis [26]. Recently, human tumorassociated chromosomal translocation was also achieved by applying Cas9 and a pair of sgRNAs targeting two chromosomes [29]. In addition to genome editing, the CRISPR/Cas9 platform with small modifications have been successfully transformed into tools to knockdown gene expression [30,31], to create engineered transcription activator or repressor [32,33], and more recently, to visualize repetitive elements in telomeres and coding genes in living cells [34].

As a novel tool for genome editing, the specificity of CRISPR/Cas9 received increasing attentions, as it is critically essential for future therapeutic applications and biological studies. Recent studies reported obvious off-target effects of CRISPR/Cas9 occurred in human cell lines [3537], whereas, other studies with mouse embryos, mouse embryonic stem cell and $X$. tropicalis embryos suggested that CRISPR/Cas9 induced off-target effect was at a low rate $[27,38,39]$.

D10A is a Cas9 nickase in which the 10th Asp in the RuvC catalytic domain is converted to Ala. Using D10A together with a pair of sgRNAs targeted to complementary strands of a target site could also induce indel mutations and was reported to decrease the off-target effect notably [36].

In this study, we utilized Cas9 paired with sgRNAs to disrupt genes that mainly involved in NC development in X. tropicalis embryos. We have designed 16 sgRNAs targeting 15 genes that are involved in $\mathrm{NC}$ specification, differentiation and migration. Among them, 14 sgRNAs are effective, showing the gene disruption rate from 9.3 to $57.8 \%$. We demonstrated simultaneous targeting of duplex genes in $X$. tropicalis embryos. We indicated that CRISPR/Cas9 system could induce segmental deletion and reversion in somatic cells. Although the Cas9 showed high specificity in most tested loci, we observed obvious offtarget effects when we targeted sox 9 locus. We then used Cas9 D10A nickase and a pair of sgRNAs to reduce the offtarget cleavages. Our studies shed light on the design and optimization of the CRISPR/Cas9 system for genome editing in X. tropicalis embryos, and set up a basis to study NC development using knockout $X$. tropicalis.

\section{Results}

\section{Disruption of gene involved in neural crest development by using CRISPR/Cas9}

The CRISPR/Cas9 has been proven to be effective for gene disruption (Fig. 1a). We therefore want to use this approach to generate genetic models that can be used for studying $\mathrm{NC}$ development. We designed sgRNA targeting the involved in NC development using the guideline as we previous reported [26, 40], and the schematic drawing was shown in Fig. 1b. Total 16 loci from 15 different genes were selected for targeting (Fig. 1; Additional file 1: Figure S1). We coinjected 500 pg Cas 9 mRNA and 40 pg sgRNA to obtain single gene disruption with high mutagenesis rate as we tested previously [26]. Forty-eight hours after injection, we randomly pooled five embryos injected with Cas9/sgRNA, extracted genomic DNA, amplified the targeted region, analyzed cleavage efficiency with T7E1 assay. The representative T7E1 gel images and calculated disruption efficiency were shown in Fig. 1c and d, respectively. We found that 


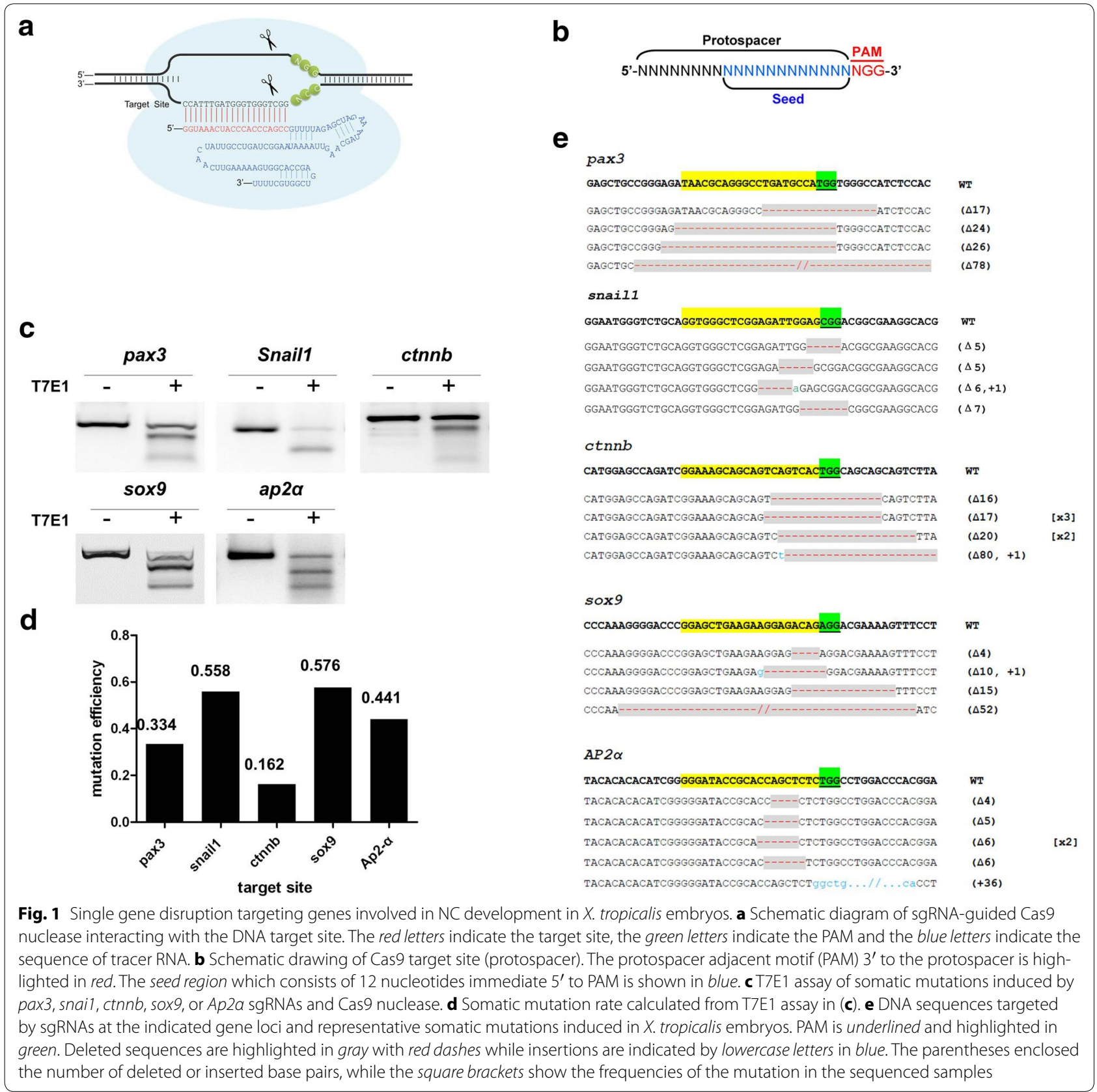

Cas9 can efficiently induce gene disruption, resulting in the cleavage efficiency from 9.3 to $57.8 \%$. The Cas9-induced indel mutations were confirmed by sequencing (Fig. 1e; Additional file 1: Figure S1).

\section{Representative phenotypes induced by CRISPR/Cas9 in G0 frogs}

High disruption efficiency could imply the knockout phenotype even at G0 frog $[9,41]$. Disruption of pax3 caused the pigmentation defects at limbs revealed by lack of pigments and more transparent skin ( 7 out of 7 ), and severe defects of paralyzed upper limbs (3 in 7), which resembled some symptoms of Waardenburg syndrome in human [42] (Additional file 1: Figure S2A-D). Another example is the disruption of tyrosinase [43, 44]. The Tyrosinase converts tyrosine into pigment melanin, therefore disruption of tyr in $X$. tropicalis embryo will cause loss of pigments in larvae, which can be easily discerned. The partial albinism was also observed in adult G0 frogs (3 in 3) (Additional file 1: Figure S2E, F). 


\section{Duplex gene disruptions induced by Cas 9}

A biological process usually was controlled by a group of genes. Loss-of-function may not be achieved by just disruption of a single gene as redundant members from the same gene family may exert complimentary function. Therefore multiplex gene disruption is valuable for studying the hierarchy of a gene regulatory network. During the NC specification, Pax3 and Zic1 coordinate to induce expression of $\mathrm{NC}$ specific transcription factors. We injected mixture of pax3 sgRNA, zic1 sgRNA and Cas 9 mRNA into $X$. tropicalis embryos. At $48 \mathrm{hpf}$, genomic DNA was extract from single embryo, and the locus specific PCR detected that mutations were induced at both pax 3 and zic1 loci in 10 out of 10 embryos. T7E1 assay indicated mutation rates were $5-23 \%$ for pax3, and $0.9-$ $32 \%$ for zic1. Sequencing data confirmed the somatic mutations induced by duplex sgRNAs (Fig. 2a-c).

We targeted snaill and snail2 simultaneously with the same strategy. Again we found both snail1 and snail2 were disrupted in single embryos. Five out of five embryos harboring mutation at snaill and snail2 loci, with mutation rate $3-40.5 \%$ for snaill and $1-7.4 \%$ for snail 2 revealed by T7E1 assay. It is notable that mutagenesis rates calculated from sequencing data were higher than those obtained from T7E1 assay. Eight out of 20 and five out of 20 colonies derived DNA from embryo \#4 harbor mutation in the loci pax3 and zic1, respectively. Similarly, Sequencing results indicated higher mutagenesis rate for snail2. Three out of 19 colonies derived from embryo \#2 harbored indel mutations (Fig. 2d-f).

\section{Targeted segmental deletion and inversions}

Although CRISPR/Cas9 with a single sgRNA could induce indel-mutations with high frequency, such indelmutations are often small $(<30 \mathrm{bp})$. This particular feature cannot satisfy requirements where larger and more predictable genomic alterations are desired. Generation of large segmental deletion is very useful to study the functions of noncoding elements, such as lincRNA, miRNA clusters and cis-regulatory modules. It is also valuable to make animal models for the diseases caused by the segmental deletions [45] or by chromosomal inversions [46]. The ability to create large segmental deletion or inversion is therefore very useful for generating animal models or cell lines for studying human diseases. To test whether CRISPR/Cas9 can efficiently induce segmental deletion or inversion in Xenopus embryos, we designed another sgRNA that targeted to $3^{\prime}$ UTR in pax3, pax3 T2 sgRNA, which is approximate $40 \mathrm{~kb}$ downstream of the pax3 sgRNA (hereafter pax3 T1 sgRNA) targeting site (Fig. 3a). We injected the mixture of pax3 T1 sgRNA, pax3 T2 sgRNA and Cas 9 into embryos at one-cell stage. Ten injected embryos were pooled and genomic DNA was extracted at $48 \mathrm{hpf}$. The possible large segmental deletions were detected by PCRs with primers that can bridge two targeting sites (Fig. 3b). The amplicons were subcloned to TA vector, and the mutations were confirmed by sequencing (Fig. 3c). The generation of inversion induced by this pair of sgRNAs was also detected by PCR by using two forward primers from each targeting sites separately (Fig. $3 \mathrm{~b}$ ) and confirmed by afterwards sequencing (Fig. 3c). Similarly, we also created segmental deletion and inversion at snaill loci using a pair of sgRNAs which target snail1 $5^{\prime}$ UTR and exon 3 respectively (hereafter snail1 T1 and snail1 T2 sgRNAs). The segment between the two snail sgRNA targeting sites is $2.5 \mathrm{~kb}$. Co-injection of snail T1, snail1 T2 sgRNAs and Cas 9 also induced segmental deletion and inversion revealed by PCR assay, and then confirmed by sequencing (Fig. 3d). Thus CRISPR/Cas9 system is an effective tool to generate segmental deletions or inversions in Хenopus embryos, which set a basis for generating Xenopus models for diseases caused by segmental deletion or inversion.

\section{Gene disruption with sgRNA designed with 5'-NAG-3' PAM sequence}

A recent study that investigated gene regulation mediated by engineered Cas 9 transcriptional activator suggested that Cas 9 can also use $5^{\prime}$-NAG- $3^{\prime}$ as an alternative PAM [33]. To test whether it is feasible for Cas9-mediated gene disruption in $X$. tropicalis embryos, we designed three sgRNAs targeting id3, myosinX, and twist 1 immediately followed by $5^{\prime}$ - NAG-3', respectively. We tested their efficacy in $X$. tropicalis embryos by injecting 40 pg NAG sgRNA with $500 \mathrm{pg}$ Cas 9 mRNA into embryos at onecell stage. As revealed by T7E1, these targeting induced mutagenesis efficiencies at $1.2 \%$ for $i d 3,3.9 \%$ for myosin $X$, and $0 \%$ for twist1 (Additional file 1: Figure S3). We amplified the target regions, TA cloned the amplicons, and sequenced the colonies for each of gene. None indel mutations were identified, which confirmed our observation with T7E1 assay. The low mutagenesis rates suggest that the $5^{\prime}$ - NAG- $3^{\prime}$ is not an effective PAM sequence for Cas9 in X. tropicalis embryos.

\section{Assess the potential CRISPR/Cas9 off-target effects in Xenopus embryos}

Although CRISPR/Cas9 platform is an effective tool for genome editing, some studies with cultured cell lines indicated obvious off-targets of CRISPR/Cas9 at some loci [35-37]. However, other studies in mouse embryonic stem cells or $X$. tropicalis embryos suggested high specificities of CRISPR/Cas9 at the tested loci [38, 39]. The CRISPR/Cas9 mediated specific DNA cleavage can be significantly reduced by single mismatch at the sgRNA target sites [11, 47], especially in the seed region (12 
a

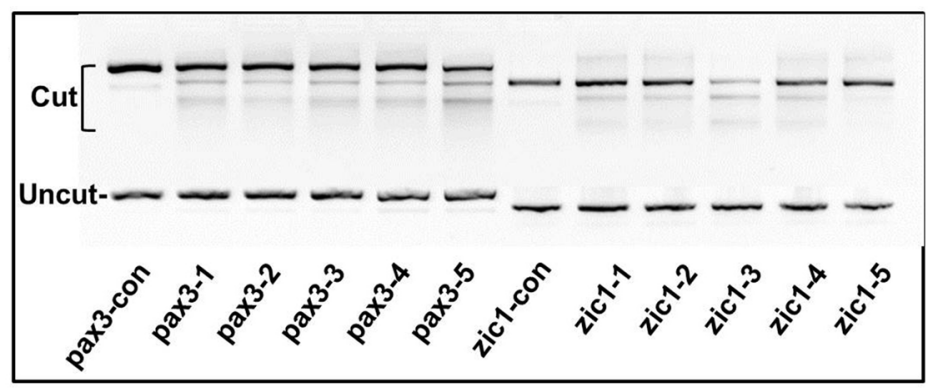

b

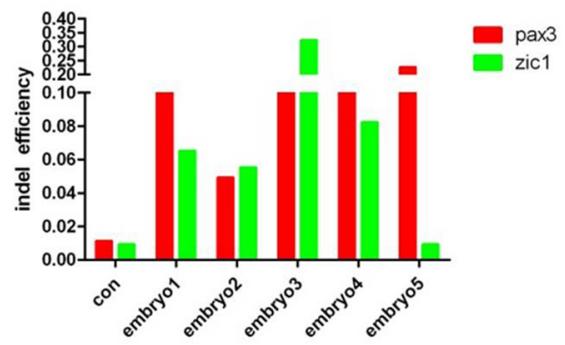

C pax 3 indel mutations in embryo 4

GAAACCCTGAGCTGCCGGGAGATAACGCAGGGCCTGATGCCATGGTGGCCATCTCCACTATCTTGT GAAACCCTGAGCTGCCGGGAGATAACGCAGGGCCTGATGgtCATGGTGGGCCATCTCCACTATCTTG GAAACCCTGAGCTGCCGGGAGATAACGCAGGGCCTGA-----TGGTGGGCCATCTCCACTATCTTGT GAAACCCTGAGCTGCCGGGAGATAACGCAGGGCC------ATGGTGGGCCATCTCCACTATCTTGT GAAACCCTGAGCTGCCGGGAGATAACC----_-----ATGGTGGGCCATCTCCACTATCTTGT GAAACCCTGAGCTGCCGGGAG----------------ATGGTGGGCCATCTCCACTATCTTGT GAAACCCTGAGCTGCC-----------------------ATGGTGGGCCATCTCCACTATCTTGT

\section{WT}

$(\Delta 1,+2)$

$(\Delta 5)$

$(\Delta 7)$

$(\Delta 14)$

$(\Delta 20)$

$(\Delta 25)$

\section{zic1 indel mutations in embryo 4}

\begin{tabular}{|c|c|c|}
\hline СTCCCAATGTGGTGAACGGACAGATGAGGCTGGGCTTCTCTGGGGACATGTATGGCAGGCCCGATCA & WT & \\
\hline CTCCCAATGTGGTGAACGGACAGATGAGGCTGGGCTTC-aTGGGGACATGTATGGCAGGCCCGATCA & $(\Delta 2,+1)$ & \\
\hline СTCCCAATGTGGTGAACGGACAGATGAGGCTGGGCTTC--TGGGGACATGTATGGCAGGCCCGATCA & $(\Delta 2)$ & [x2] \\
\hline CTCCCAATGTGGTGAACGGACAGATGAGGCTGGGCT-----GGGGACATGTATGGCAGGCCCGATCA & $(\Delta 5)$ & \\
\hline CTCCCAATGTGGTG & $(\Delta \mathbf{3 0})$ & \\
\hline
\end{tabular}

d

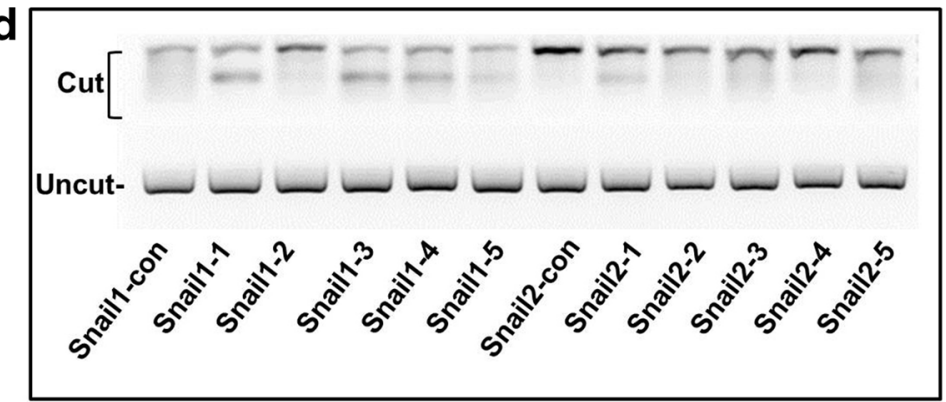

e

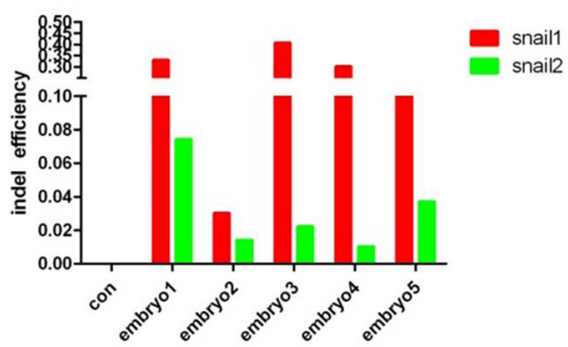

snail1 indel mutations in embryo 1

TTGACGTCGGAATGGGTCTGCAGGTGGGCTCGGAGATTGGAGCGGTCGGCGAAGGCACGGTTGCAGT

TTGACGTCGGAATGGGTCTGCAGGTGGGCTCGGAGATTGG--tGGTCGGCGAAGGCACGGTTGCAGT TTGACGTCGGAATGGGTCTGCAGGTGGGCTCGGAGAGCGG-----TCGGCGAAGGCACGGTTGCAGT TTGACGTCGGAATGGGTCTGCAGGTGGGCTCGGAGCG-------GTCGGCGAAGGCACGGTTGCAGT GACGTCGGAATGGGTCTGCAGGTGGGCTCGGtc $g$ gcggt c 9 GCGGTCGGCGAAGGCACGGTTGCAGT TTGACGTCGGAATGGGTCTGCAGGTGAGCGGTCGGCG-..-_..--.-AAGGCACGGTTGCAGT

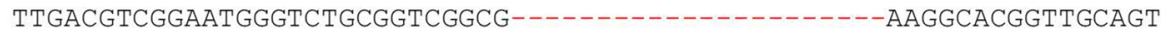

WT

$(\Delta 3,+1)$

$(\Delta 5)$

$(\Delta 7)$

$(\Delta 10,+7) \quad[x 2]$

$(\Delta 14)$

$(\Delta 22)$

[x2]

\section{snail2 indel mutations in embryo 1}

\begin{tabular}{|c|c|}
\hline СGTCTAAAGACCACAGTGGCTCGGAGAGCCCCATCAGCGATGAGGAGGAAAGACTCCAAACCAAACT & WT \\
\hline CGTCTAAAGACCACAGTGGCTCGGAGAGCCCCATCA----TGAGGAGGAAAGACTCCAAACC & $(\Delta 4)$ \\
\hline CGTCTAAAGACCACAGTGGCTCGGAGAGCCCCATCAGCG----GGAGGA & $(\Delta 4)$ \\
\hline CGTCTAAAGACCACAGTGGCTCGGAGAGCCCCATCAtC----------- & $(\Delta 14,+2)$ \\
\hline
\end{tabular}

Fig. 2 Duplex indel mutations in single embryo. a, d T7E1 assay of duplex indel mutations in the loci of pax3 and zic1 (a) or snail1 and snail2 (d) in five single embryos. b, e Quantification of the T7E1 assay in (a) or (d). c, f Representative sequencing data confirmed the indel mutations induced at the two target loci in one single embryo 
a

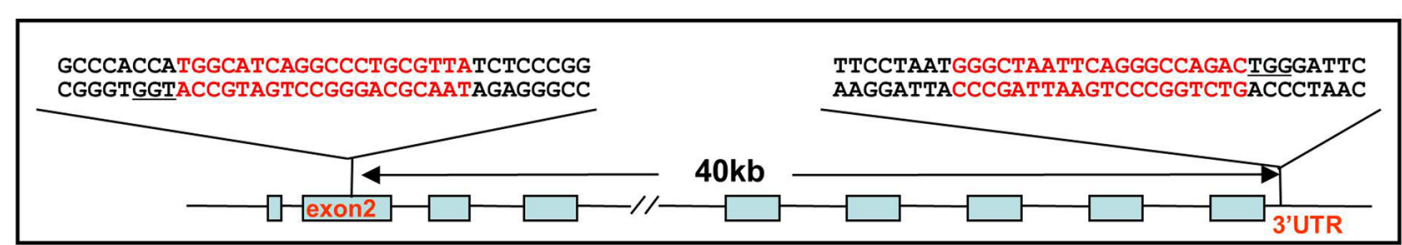

Targeting sites at pax3 locus for generating segmental deletion/inversion

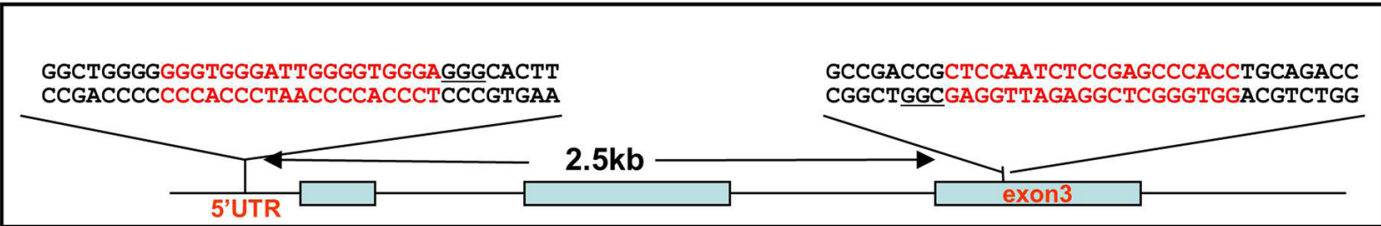

Targeting sites at snail1 locus for generating segmental deletion/inversions

b
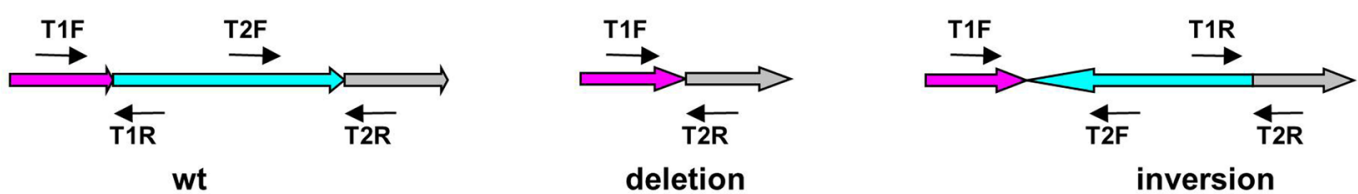

C

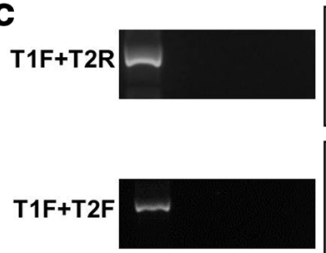

TGGCCCACCATGGCATCAGGCCCTGCGTTA $\ldots 40 \mathrm{~kb} \ldots$ GGGCTAATTCAGGGCCAGACTGGGATT

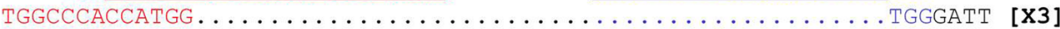

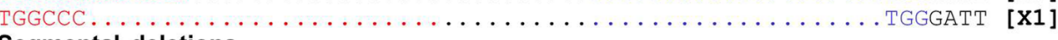

Segmental deletions

GGAGATGGCCCACCATGGCATCAGGCCTGCGTTAGTCTGGCCCTGAATTAGCCCATTAGGAAAATT

GGAGATGGCCCACCATGG ................. ThGCCCTGAATTAGCCCATTAGGAAAATT [x $[\mathbf{x}]$

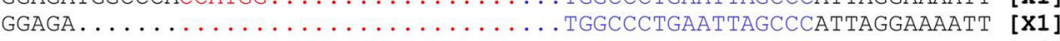

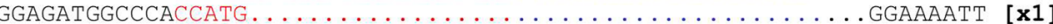

Segmental inversions (sequences at $5^{\prime}$ junction)

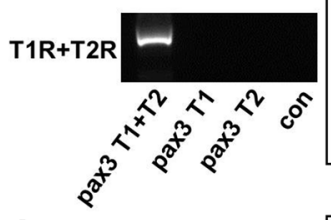

d

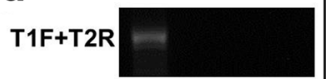

GCTGCCGGGAGATAACGCAGGGCCTGATGCCATGGGGGCTAATTCAGGGCCAGACTGGGATTCAAAA

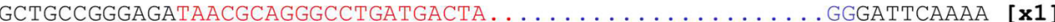

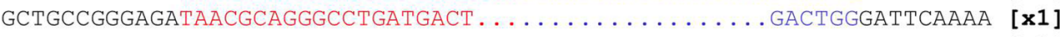

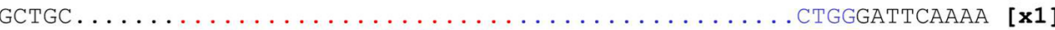

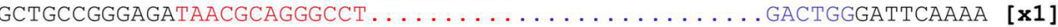

Segmental inversions (sequences at $3^{\prime}$ junction)

CTGGGGGGGTGGGATTGGGGTGGG $\ldots 2.5 \mathrm{~kb} \ldots \ldots \ldots$ CCGCTCCAATCTCCGAGCCCACCTGCA

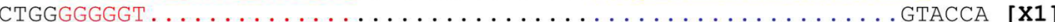

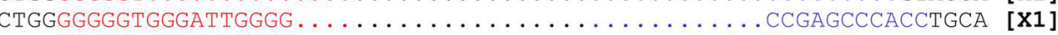

CTGGGGGGTGGGATTGGGGT . . . . . . .

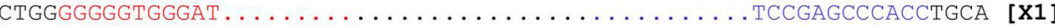

Segmental deletions

GGGGGCTGGCTGGGGGGGTGGGATTGGGGTGGGGGTGGGCTCGGAGATTGGAGCGGTCGGCGAAGCG

GGGGGCTGGCTGGGGGGGTGGGATTGGGGTAGGG . . . 155bp ... TTGGCTTCGGTCGGCGAAGCG [X1]

GGGGGCTGGCTGGGGGGGTGGGATTGG $\ldots \ldots \ldots \ldots \ldots \ldots \ldots \ldots \ldots \ldots \ldots \ldots$ GGCGAGCG $[\mathbf{x} 2]$

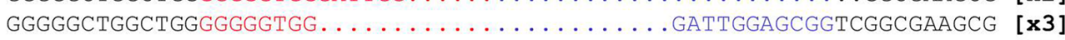
GGGGGCTGGCTGGGGGGGTGGGATTGGG ...............................

Segmental inversions (sequences at 5 ' junction)

TGTGGGCAAAGTGCCCTCCCACCCCAATCCCACCCCAATCTCCGAGCCCACCTGCAGACCCATTCCG

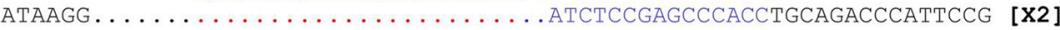

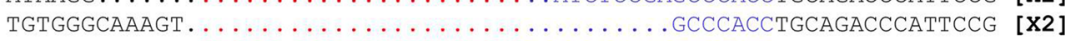

. ATCTCCGAGCCCACCTGCAGACCCATTCCG [X4]

Segmental inversions (sequences at $3^{\prime}$ junction) 
(See figure on previous page.)

Fig. 3 Segmental deletions and inversions induced by Cas9 and a pair of sgRNAs in X. tropicalis embryos. a Schematic diagram showing the structure of $X$. tropicalis pax3 and snail1 genes, and the sgRNA targeting sequences and their locations in the two genes. b Schematic diagram illustrating detection of segmental deletion and inversion at targeted loci with PCR. $\mathbf{c}, \mathbf{d}$ PCR assay and subsequent sequencing detected segmental deletions and inversion at pax3 locus (c) and snail l locus (d). The embryos injected with Cas 9 and indicated sgRNAs were collected at 2 days after fertilization. Five embryos were pooled for DNA extraction. T1, embryos injected with Cas 9 mRNA and sgRNA T1; T2, embryos injected with Cas 9 mRNA and sgRNA T2; T1 + T2, embryos injected with mixture of Cas9 mRNA, sgRNA T1 and sgRNA T2. F forward primer; R reverse primer

nucleotides located in the $3^{\prime}$ next to PAM, Fig. 1b). We also scanned $X$. tropicalis genome to identify potential off-target sites for the 16 targeted loci, and found the potential off-target sites corresponding to 11 target loci (Additional file 1: Table S3). These potential off-target loci from the targeted embryos were amplified by locus specific PCR, and the amplicons were analyzed by T7E1 assay. Totally, 25 potential off-target loci corresponding to the 11 target loci were analyzed by T7E1 assay, and 11 off-target loci showed T7E1 positive (Fig. 4). We selected the loci with over $10 \%$ mutagenesis rate, and subcloned the amplicons into PMD-18T vectors. Five to eleven bacterial colonies for each off-target loci were randomly picked for sequencing. Two potential off-target sites, sox9-579 and lrig3-E306 were failed in T7E1 analysis, since no clear and specific bands after T7E1 assay were detected due to repetitive sequences in the region. Although T7E1 assay suggested obvious off-target mutagenesis at Ap2 $\alpha-963$ (50\%), sox9-826 (15.4\%), and zic1-645 (32\%) loci, sequencing data did not reveal
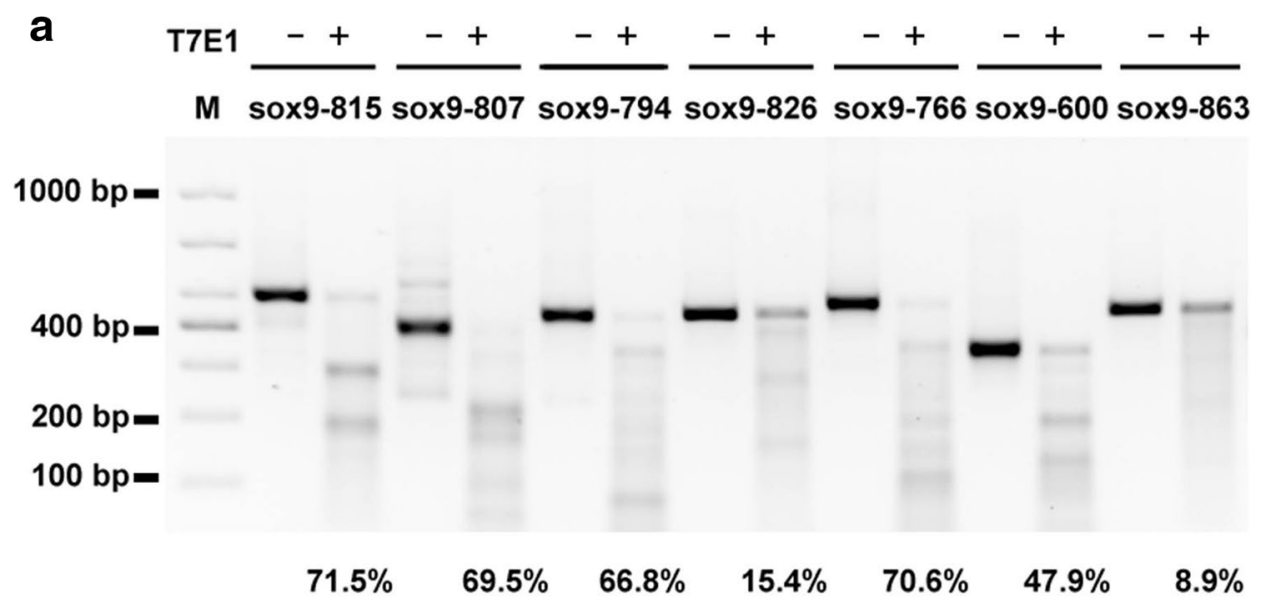

b

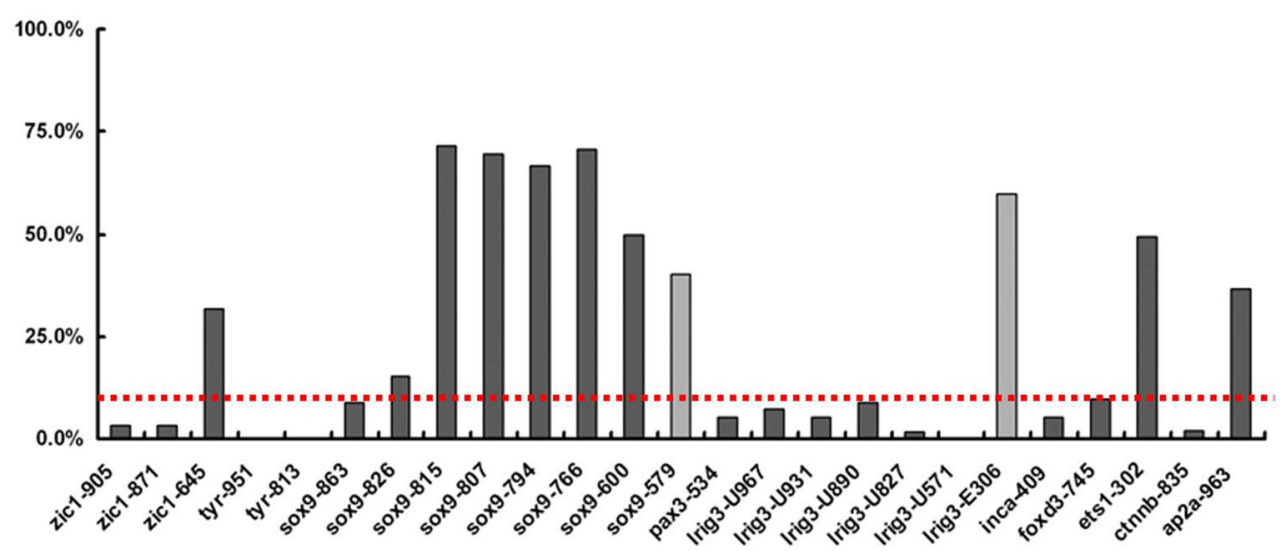

Fig. 4 Off-target effects of CRISPR/Cas9 in X. tropicalis evaluated via T7E1 assay. a Representative gel image of T7E1 digested off-target amplicons. The percentage under panels indicated the frequencies of off-target effects. $\mathbf{b}$ Quantification of the cleavage frequencies of all off-target sites. No clear and specific bands after T7E1 assay were generated at sox9-579 and Irig3-E306 loci. The cutting efficiencies at sox9-579 and Irig3-E306 sites were calculated by sequencing results. Red dash line indicated $10 \%$ in indels efficiency 
any mutations at these loci (Additional file 1: Figure S4). However, off-target mutagenesis induced at other examined loci was profound. Among them, mutations were found in all the sequenced samples at five examined loci (sox9-600, sox9-766, sox9-794, sox9-807, and sox9-815). The sequence variations occurred at ets1-302 were proven as nucleotide polymorphism because the uninjected embryos also harbors the same DNA alternations (Additional file 1: Figure S4). Collectively, these results indicated that CRISPR/Cas9 could induce obvious mutagenesis at some off-target loci in $X$. tropicalis embryos.

\section{D10A nickase reduced off-target cleavage in Xenopus embryos}

Recent reports indicated the off-target mutations induced by Cas 9 in cultured cell lines [11,35-37]. We also observed substantial off-target effects when we used Cas 9 to target sox 9 locus. Since the high specificity is essential for genome editing, we next sought to find approaches to minimize off-target effect of CRISPR/ Cas9. The two nuclease domains in Cas9, HNH and RuvC, cleave the DNA strand complementary and noncomplementary to the sgRNA, respectively. Point mutation of D10A in RuvC or H840A in HNH converted Cas9 into DNA nickases $[11,12]$. Recent studies with cultured mammalian cells indicated that off-target effects can be significantly reduced by using D10A nickase with a pair of sgRNAs targeting both strands [36]. We therefore test the efficiency of this approach in $X$. tropicalis embryos at pax3 and ets 1 loci. First, we tested whether the D10A approach can induce gene disruption in $X$. tropicalis embryos. We designed a sgRNA against pax3 at the $5^{\prime}$ upstream of the previous target site ( a $3 \times 3$ T3 sgRNA). The offset of the sgRNA pair (pax3 T1 and pax3 T3) is $+5 \mathrm{bp}$, while the $5^{\prime}$ overhang is $42 \mathrm{bp}$ (Additional file 1 : Figure S5A). We injected D10A Cas9 mRNA together with pax3 sgRNA pairs into the $X$. tropicalis embryos at stage one and detected the mutation efficiency by direct sequencing (Additional file 1: Figure S5B). The mutation efficiency induced by nickase was comparable to wild type Cas9 (36.8 vs $47.1 \%)$. Similarly, we designed another sgRNA targeting ets1 (ets1 T2). The offset of sgRNA pairs is +64 bp. Co-injection of Cas 9 mRNA and the pair of ets1 sgRNAs induced indel mutations as well with ratio of $5.3 \%$ (Additional file 1: Figure S5C, D).

The sox 9 sgRNA/Cas 9 can induced indel mutation at sox 9 locus as well as the off-target sites at sox9-600, sox9766, sox9-794, sox9-807, and sox9-815 (Fig. 4; Additional file 1: Figure S4). We designed another sgRNA targeting sox 9 locus at 9 bp $5^{\prime}$ upstream to the sox 9 sgRNA targeting site ( $\operatorname{sox} 9 \mathrm{~T} 2$ ), both of which will generate $43 \mathrm{bp} 5^{\prime}$ overhang (Fig. 5a).
We injected D10A mRNA together with sox 9 T1 and sox 9 T2 sgRNAs into $X$. tropicalis embryos, and examined the on-target indel mutation by T7E 1 and direct sequencing. The indel mutation ratio is $32.6 \%$ (T7E1 data not shown), which is lower than we obtained with wild type Cas9/sox9 T1 sgRNA (Fig. 5b). We next examined the effects of this configuration on the off-target sites. Revealed by direct sequencing, the ratio of mutation at sox9-600 locus was reduced from 50 to $10 \%$, suggesting D10A nickase can reduce the off-target efficiency in X. tropicalis (Fig. 5c-f).

\section{Cas9-induced mutagenesis is highly heritable}

The Cas9 targeted embryos were raised to sexual maturity, and crossed with wild type frogs. We collected embryos, extracted DNA from single embryos and used T7E1 assay to determine whether the embryos harbored mutations. T7E1 assay indicated that one embryo from one founder male frog of $z i c 1$, and three embryos from two founder male frogs of pax3, snaill and id3, respectively, carried the induced mutations. Such mutations were further confirmed by sequencing. So the rate of germ line transmission of the mutations ranged from 5 to $22 \%$ (Additional file 1: Figure S5). The data again indicate that sgRNA/Cas9-induced mutagenesis is highly heritable. We are in the progress of establishing the knockout lines using the approach we described previously [48].

\section{Discussion}

In this study, we utilized CRISPR/Cas9 platform to disrupt genes involved in NC development. In addition to the single gene disruption, we also successfully generated duplex and segmental gene disruptions. After evaluation of off-target effects in $X$. tropicalis embryos, we found that the Cas9 D10A nickase approach can reduce offtarget cleavage. Our studies add versatility to this powerful tool for genome editing in $X$. tropicalis embryos. With improving the specificity, this simple and highly versatile tool will greatly expand our capacity on biological researches.

Currently, most laboratories utilize Cas9 from S. pyogenes for genome editing, and select $5^{\prime}-\mathrm{N}_{20}-\mathrm{NGG}-3^{\prime}$ sequence as its targeting sites (Fig. 1b). Based on this finding, the chance for designing sgRNA is $1 / 8$ in a given genome. We have successfully targeted 14 genes that involved in $\mathrm{NC}$ formation, migration and differentiation using this approach, and will continue to establish knockout $X$. tropicalis lines step by step. We observed some phenotypes from G0 frogs and showed the representatives in Additional file 1: Figure S2. It should be mentioned that the G0 frogs are mosaic, and G0 phenotypes could lead to adverse effects to their progeny as well. The G0 frog phenotype may provide some hints for the gene function. The 


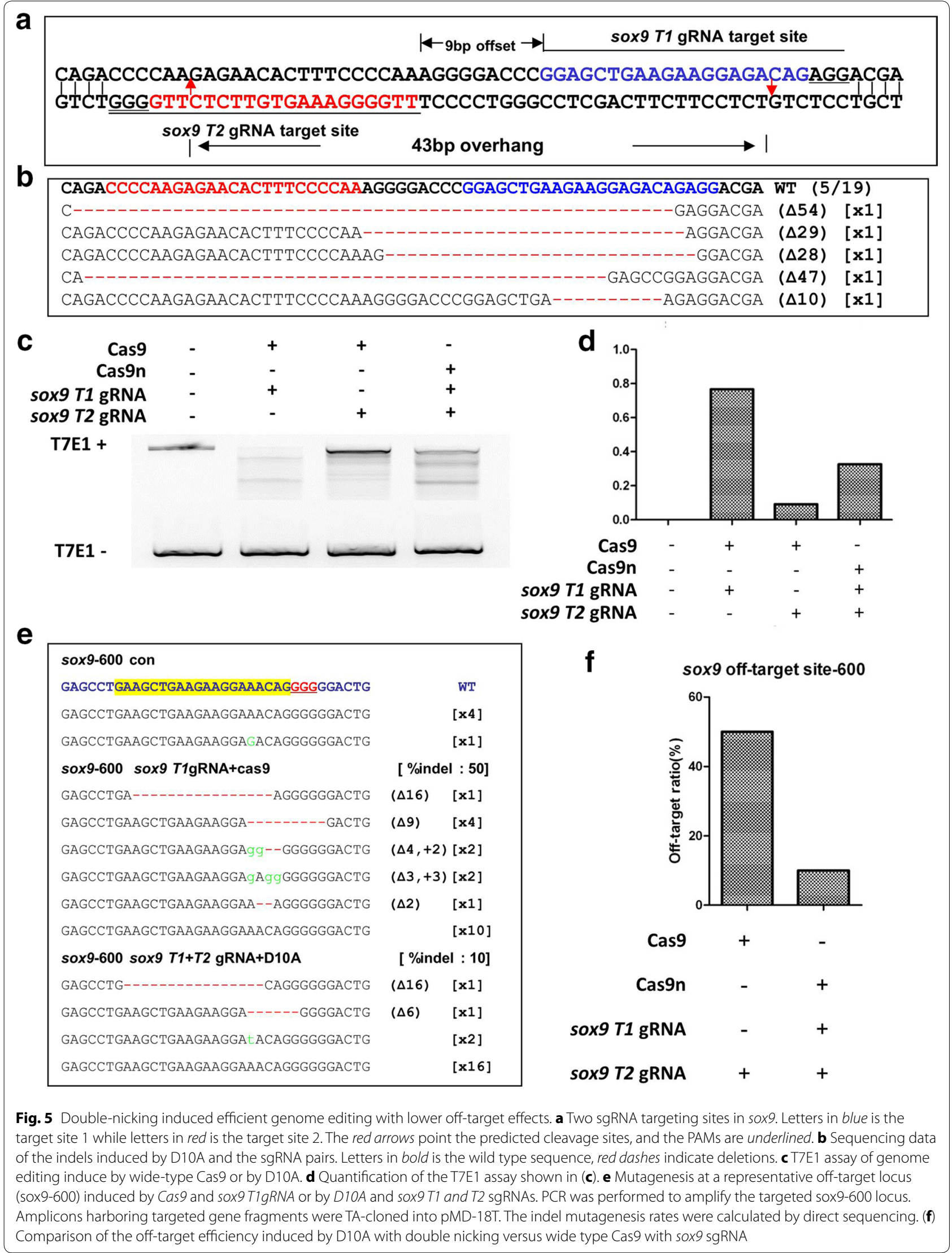


gene knockout phenotype can only be determined after the knockout line is established, and we should know and distinguish these two kinds of phenotypes.

In addition, recent report suggested that $5^{\prime}$-NAG- $3^{\prime}$ can also serve as PAM for Cas9. We tested three targeted sites that were followed by NAG at twist1, myosin $X$ and id3 loci, respectively, none of them can be disrupted (Additional file 1: Figure S3). Our data suggested that NAG cannot serve as an effective PAM for Cas9, at least in X. tropicalis embryos.

Multiplex mutations at different loci can be simultaneously induced in one embryo by co-injection of Cas 9 mRNA and a combination of corresponding sgRNAs [26]. The NC specification was regulated by combined effects of pax3 and zic1 [49]. The two transcription factors of snail1 and snail2 play important roles in epithelial-mesenchymal transition of NC development [50,51]. We used Cas 9 induced duplex somatic mutations at pax3 and zic1 loci, and snail1 and snail2 loci in X. tropicalis embryos (Fig. 2). Generation of large segmental deletion is very useful for studying the function of non-coding DNA sequences or regulatory DNA elements. It can be also used for deletion of a particular protein domain to study gene functions. Our data indicated that CRIPSR/ Cas9 is efficient to generate large segmental deletion/ inversions. Such abilities make CRISPR/Cas9 system a very useful platform for functional studies and for generation of diseases models.

In our pilot gene disruption studies with CRISPR/Cas9, we did not identified obvious off-target effects at some gene loci [26]. However, with increasing number of gene loci tested by CRISPR/Cas9, we found off-target in some gene loci (Fig. 4 and Additional file 1: Figure S4). Most off-target loci we identified are located in non-coding region and even intergenic region, which may not affect normal development even indel mutations were induced in these regions. We suggest $10 \%$ T7E1 rate as a cut-off for further evaluation of the off-target cleavages. The off-target cleavages with low mutagenesis rate could be diluted through backcross with wild type animals.

The paired D10A approach can be a good solution to minimize the potential off-target effects induced by Cas9. In line with previous research work, we also observed the off-cleavages were reduced about five times when we targeted sox 9 locus with D10A and a pair of sgRNAs (sox 9 $T 1+T 2$ sgRNAs) compared to those induced by wild type Cas9 and sox9 sgRNA (Fig. 5). The design of sgRNA pairs followed the principles described previously. Briefly, the offsets between the sgRNA pairs are less than $100 \mathrm{bp}$, and ideally from -4 to $20 \mathrm{bp}$; and the sgRNA pair should generate a $5^{\prime}$ overhang other than $3^{\prime}$ overhang. The sgRNA pairs fulfill the above conditions may generate indel mutants.
Our criteria for identification of potential off-target sites seem reliable. A previous report indicated that even two mismatches to sgRNA were well tolerated by Cas9 to induce DNA cleavages at some loci, while three or more mismatches can significantly reduce the mutagenesis capacity [35]. Our criteria allow three mismatches, which could cover most of the potential off-target sites.

A few strategies may reduce the potential off-target cleavage induced by Cas9. (1) Use Cas9 D10A nickase coupled with a pair of sgRNAs to reduce potential offtargets. (2) Reduce injection doses of Cas9 mRNA and sgRNA which may decrease the possible mis-binding of sgRNA to the off-target sites, or if possible, select another sgRNA binding site. A study suggests that the off-target effects occur as sequence dependent [52]. (3) Perform BLAST search, and avoid homologous sequences to reduce the possible off-target binding of gRNAs [53]. (4) Utilize the dCas9-FokI approach that was reported to reduce the off-target cleavage [54].

Taken together, we used CRISPR/Cas9 to induce indel mutations at gene loci involved in NC development in $X$. tropicalis, and set a basis for studying this developmental process using genetic animal models. When compared with the other two genome editing tools, ZFN and TALEN, the CRISPR/Cas9 platform is simpler and more convenient. Modified CRISPR/Cas9 platform with high specificity will have great impacts on biomedical research and treating human diseases.

\section{Conclusion}

The NC cells are highly pluripotent that can differentiate into a large variety of cell derivatives. Many congenital birth defects are due to abnormalities of NC development. While Xenopus has been utilized as an ideal model to study the NC development, currently loss-of-function assay in Xenopus embryos are largely based on the MOs knockdown approach, which is hurdled by its transient effects. In this study, we took advantages of CRISPR/Cas9 system to induce targeted gene mutations in X. tropicalis embryos. We designed sgRNAs targeting 16 loci in 15 genes, 14 of which could induce indel mutations with relatively high efficiency. We generated duplex mutations and large fragment deletions or inversions using this system as well. The off-target effects of this system in X. tropicalis embryos was carefully evaluated, and we showed evidence that the Cas9 D10A nickase approach can reduce off-target cleavage.

Our studies proved CRISPR/Cas9 system as a powerful genome editing tool in $X$. tropicalis embryos. By crossing with the wild type frogs, we obtained the G1 heterozygotes harboring mutations. We can utilize this platform to study the functions of the genes involved in NC development in future. 


\section{Methods}

All experimental protocols were approved by The Government of the Hong Kong Special Administrative Region, Department of Health. The methods were carried out in accordance with the approved guidelines.

\section{$X$. tropicalis maintenance and husbandry}

The $X$. tropicalis was purchased from Nasco Co. (USA), and the frog maintenance in principle followed the Harland $X$. tropicalis Website (http://tropicalis.berkeley.edu/ home/). Synchronized embryos were obtained by in vitro fertilization using method describe previously $[9,48]$.

\section{DNA constructs}

The human codon-usage optimized $\operatorname{Cas} 9$ constructs were created by Church's lab and purchased from Addgene (Cat. No. 41815). pCS2-Cas9-NLS was generated by inserting Cas9 ORF coding sequence and relative SV40 T-antigen nuclear location signal (NLS) into $p C S 2+$ vector. The pCS2-Cas9-2NLS was constructed by inserting two NLS together with Cas9 coding sequence [26].

The sgRNA sequences were designed by using webbased program ZiFiT Targeter Version 4.2 (http://zifit. partners.org/ZiFiT/). Two oligonucleotides with the targeting sequence containing Bsal cutting sites at $5^{\prime}$ end were annealed first, and the annealed oligonucleotides were cloned into sgRNA vector pDR274 (Addgene \#42250) [55] after BsaI digestion. The oligo sequences for sgRNA constructs were shown in Additional file 1: Table S1.

\section{Preparation of Cas9 mRNA and sgRNA for microinjection} The pCS2-Cas9-NLS and pCS2-Cas9-2NLS were linearized by NotI (NEB), respectively. Cas 9 mRNAs were transcribed with mMESSAGE mMACHINE SP6 Kit (Life Technologies, Ambion) using linearized Cas 9 constructs as template. The Cas 9 mRNAs were purified using RNA purification kit (QIAgen) and eluted in RNase-free water. The sgRNAs were transcribed with T7 RNA polymerase after linearization of sgRNA constructs by DraI, and purified with mirVana miRNA Isolation kit (Life Technologies, Ambion).

\section{Microinjection}

The mixture of Cas 9 mRNA and sgRNAs as indicated in the figures were injected into $X$. tropicalis embryos at one-cell stage. Microinjection was followed as previous studies $[9,48]$. The injected embryos were collected for DNA extraction at $48 \mathrm{~h}$ after fertilization.

\section{T7 endonuclease 1 (T7E1) assay}

T7E1 assay was basically performed as previously described [26, 56]. Briefly, 150-200 ng amplicons were denatured and re-annealed in $19 \mu \mathrm{l}$ digestion solution using the following program: $95{ }^{\circ} \mathrm{C}$ for $5 \mathrm{~min}, 95-85^{\circ} \mathrm{C}$ at $-2{ }^{\circ} \mathrm{C} / \mathrm{s}, 85-25^{\circ} \mathrm{C}$ at $-0.05{ }^{\circ} \mathrm{C} / \mathrm{s}$, and then maintained at $4{ }^{\circ} \mathrm{C}$. The annealed DNA samples were digested with T7E1 (NEB M0302L), and then separated by $1.5 \%$ agarose gel. DNA was visualized by GelRed staining. DNA signals were quantified using ImageJ software (http:// rsb.info.nih.gov/ij/). The PCR primers for T7E1 assay to detect on-target cleavages were shown in Additional file 1: Table S2.

\section{Determination of mutagenesis rate}

We normally utilize T7E1 assay to determine the mutagenesis rate. However, T7E1 can give false positive at some gene loci due to the repetitive segments or nucleotide polymorphism in $X$. tropicalis genome. We used direct sequencing to determine the mutagenesis rate (colonies harboring mutations/total colonies) in case the T7E1 assay seems not reliable.

\section{Identification of potential off-target sites in X. tropicalis}

Potential off-target sites were identified in $X$. tropicalis genome based on the following criteria, (1) tolerate up to three mismatches in the sgRNA-DNA interface sequence (protospacer); (2) only allow one mismatch in the seed region (12 nucleotides $5^{\prime}$ to the PAM); (3) PAM is the $5^{\prime}$-NGG-3' motif and the $\mathrm{N}$ represents four nucleotides. The potential off-target sites were amplified by PCR using genomic DNA extracted from Cas9/sgRNA injected embryos. Amplicons were either digested by T7E1 assay or subcloned into TA cloning vector for sequencing. The off-target cleavages were confirmed by DNA sequencing. The PCR primers for T7E1 assay to detect off-target cleavages were shown in Additional file 1: Table S4.

\section{Additional file}

Additional file 1. Supplementary figures and legends.

\section{Authors' contributions}

ZL, TTKC, ZS, ZL, YL, CW, and WS performed experiments. XC did bioinformatics analysis. $X Q, D C, B F$, and $Y D$ provided analytical tools. $H Z$ and $Y C$ conceived, ordinated and supervised this study. ZH, YD, and YC wrote the manuscript. All authors read and approved the final manuscript.

\footnotetext{
Author details

${ }^{1}$ Key Laboratory for Regenerative Medicine, Ministry of Education, School of Biomedical Sciences, Faculty of Medicine, The Chinese University of Hong Kong, Hong Kong, SAR, China. ${ }^{2}$ Shenzhen Key Laboratory of Cell Microenvironment, Department of Biology, South University of Science and Technology of China, Shenzhen, Guangdong 518055, China. ${ }^{3}$ Advanced Biomedical Computing Center, National Cancer Institute, National Institutes of Health, Frederick, MD 21702, USA. ${ }^{4}$ Key Laboratory for Regenerative Medicine of Ministry of Education, Ji Nan University, Guangzhou 510632, China. ${ }^{5}$ KIZ-CUHK Joint Laboratory of Bioresources and Molecular Research of Common Diseases, Hong Kong, SAR, China. ${ }^{6}$ Department of Developmental and Regenerative
} 
Biology, College of Life Science and Technology, Ji Nan University, Guangzhou 510632, China.

\section{Acknowledgements}

The authors thank Prof. George M Church, Feng Zhang, James A. Thomason, and Erik J. Sontheimer, and Addgene for making the plasmids available to research community. The authors are in debt to those colleagues whose work could not be cited due to space constraint. This work is supported by Grants from the Research Grants Council of Hong Kong CUHK413/12 and CUHK24100414 to HZ, by 31471367 and 31271554 of National Natural Science Foundation of China to YC, by fund of 81200566 from the National Basic Research Program of China to YD, and the Fundamental Research Funds for the Central Universities (Ji Nan University) (11614601) to HZ and XFQ. YC and YD are also supported by Shenzhen Key Laboratory of Cell Microenvironment (ZDSYS20140509142721429). ZZL and TKC are supported by the Graduate Studentships from The Chinese University of Hong Kong. We thank colleagues in our laboratories for helpful discussion on this project.

\section{Competing interests}

The authors declare that they have no competing interests.

\section{Received: 19 January 2016 Accepted: 15 March 2016} Published online: 31 March 2016

\section{References}

1. Sauka-Spengler T, Bronner-Fraser M. A gene regulatory network orchestrates neural crest formation. Nat Rev Mol Cell Biol. 2008;9(7):557-68.

2. Porteus MH, Baltimore D. Chimeric nucleases stimulate gene targeting in human cells. Science. 2003;300(5620):763.

3. Miller JC, Holmes MC, Wang J, Guschin DY, Lee YL, Rupniewski I, Beausejour CM, Waite AJ, Wang NS, Kim KA, et al. An improved zinc-finger nuclease architecture for highly specific genome editing. Nat Biotechnol. 2007;25(7):778-85.

4. Wood AJ, Lo TW, Zeitler B, Pickle CS, Ralston EJ, Lee AH, Amora R, Miller $J$ C, Leung E, Meng X, et al. Targeted genome editing across species using ZFNs and TALENs. Science. 2011;333(6040):307.

5. Christian M, Cermak T, Doyle EL, Schmidt C, Zhang F, Hummel A, Bogdanove AJ, Voytas DF. Targeting DNA double-strand breaks with TAL effector nucleases. Genetics. 2010;186(2):757-61.

6. Zhang F, Cong L, Lodato S, Kosuri S, Church GM, Arlotta P. Efficient construction of sequence-specific TAL effectors for modulating mammalian transcription. Nat Biotechnol. 2011;29(2):149-53.

7. Miller JC, Tan S, Qiao G, Barlow KA, Wang J, Xia DF, Meng X, Paschon DE, Leung E, Hinkley SJ, et al. A TALE nuclease architecture for efficient genome editing. Nat Biotechnol. 2011;29(2):143-8.

8. Boch J, Scholze H, Schornack S, Landgraf A, Hahn S, Kay S, Lahaye T, Nickstadt A, Bonas U. Breaking the code of DNA binding specificity of TAL-type III effectors. Science. 2009;326(5959):1509-12.

9. Lei Y, Guo X, Liu Y, Cao Y, Deng Y, Chen X, Cheng CH, Dawid IB, Chen Y, Zhao $H$. Efficient targeted gene disruption in Xenopus embryos using engineered transcription activator-like effector nucleases (TALENs). Proc Natl Acad Sci USA. 2012;109(43):17484-9.

10. Wiedenheft B, Sternberg SH, Doudna JA. RNA-guided genetic silencing systems in bacteria and archaea. Nature. 2012;482(7385):331-8.

11. Cong L, Ran FA, Cox D, Lin S, Barretto R, Habib N, Hsu PD, Wu X, Jiang W, Marraffini LA, et al. Multiplex genome engineering using CRISPR/Cas systems. Science. 2013;339(6121):819-23.

12. Jinek M, Chylinski K, Fonfara I, Hauer M, Doudna JA, Charpentier E. A programmable dual-RNA-guided DNA endonuclease in adaptive bacterial immunity. Science. 2012;337(6096):816-21.

13. Mali P, Yang L, Esvelt KM, Aach J, Guell M, DiCarlo JE, Norville JE, Church GM. RNA-guided human genome engineering via Cas9. Science. 2013;339(6121):823-6.

14. Mali P. RNA-guided human genome engineering via Cas9. Science. 2013;339:823-6.

15. Hwang WY. Efficient genome editing in zebrafish using a CRISPR-Cas system. Nat Biotechnol. 2013;31:227-9.
16. Yang D. Effective gene targeting in rabbits using RNA-guided Cas9 nucleases. J Mol Cell Biol. 2014;6(1):97-9.

17. Xie K, Yang Y. RNA-guided genome editing in plants using a CRISPR-Cas system. Mol Plant. 2013;6:1975-83.

18. Wang Y. The CRISPR/Cas system mediates efficient genome engineering in Bombyx mori. Cell Res. 2013;23:1414-6.

19. Wang $\mathrm{H}$. One-step generation of mice carrying mutations in multiple genes by CRISPR/Cas-mediated genome engineering. Cell. 2013;153:910-8.

20. Shan Q. Targeted genome modification of crop plants using a CRISPR-Cas system. Nat Biotechnol. 2013;31:686-8.

21. Nekrasov V, Staskawicz B, Weigel D, Jones JD, Kamoun S. Targeted mutagenesis in the model plant Nicotiana benthamiana using Cas9 RNAguided endonuclease. Nat Biotechnol. 2013;31:691-3.

22. Li JF. Multiplex and homologous recombination-mediated genome editing in Arabidopsis and Nicotiana benthamiana using guide RNA and Cas9. Nat Biotechnol. 2013;31:688-91.

23. Friedland AE. Heritable genome editing in C. elegans via a CRISPR-Cas9 system. Nat Methods. 2013;10:741-3.

24. DiCarlo JE. Genome engineering in Saccharomyces cerevisiae using CRISPR-Cas systems. Nucleic Acids Res. 2013;41:4336-43.

25. Bassett AR, Tibbit C, Ponting CP, Liu JL. Highly efficient targeted mutagenesis of Drosophila with the CRISPR/Cas9 system. Cell Reports. 2013:4:220-8

26. Guo X, Zhang T, Hu Z, Zhang Y, Shi Z, Wang Q, Cui Y, Wang F, Zhao H, Chen Y. Efficient RNA/Cas9-mediated genome editing in Xenopus tropicalis. Development. 2014;141(3):707-14.

27. Xiao A, Wang Z, Hu Y, Wu Y, Luo Z, Yang Z, Zu Y, Li W, Huang P, Tong X, et al. Chromosomal deletions and inversions mediated by TALENs and CRISPR/Cas in zebrafish. Nucleic Acids Res. 2013;41(14):e141.

28. Gupta A, Hall VL, Kok FO, Shin M, McNulty JC, Lawson ND, Wolfe SA. Targeted chromosomal deletions and inversions in zebrafish. Genome Res. 2013:23(6):1008-17.

29. Torres R, Martin MC, Garcia A, Cigudosa JC, Ramirez JC, RodriguezPerales S. Engineering human tumour-associated chromosomal translocations with the RNA-guided CRISPR-Cas9 system. Nat Commun. 2014;5:3964.

30. Larson MH, Gilbert LA, Wang X, Lim WA, Weissman JS, Qi LS. CRISPR interference (CRISPRi) for sequence-specific control of gene expression. Nat Protoc. 2013;8(11):2180-96.

31. Gilbert LA, Larson MH, Morsut L, Liu Z, Brar GA, Torres SE, Stern-Ginossar $\mathrm{N}$, Brandman O, Whitehead EH, Doudna JA, et al. CRISPR-mediated modular RNA-guided regulation of transcription in eukaryotes. Cell. 2013;154(2):442-51.

32. Cheng AW, Wang H, Yang H, Shi L, Katz Y, Theunissen TW, Rangarajan S, Shivalila CS, Dadon DB, Jaenisch R. Multiplexed activation of endogenous genes by CRISPR-on, an RNA-guided transcriptional activator system. Cell Res. 2013;23(10):1163-71.

33. Mali P, Aach J, Stranges PB, Esvelt KM, Moosburner M, Kosuri S, Yang L, Church GM. CAS9 transcriptional activators for target specificity screening and paired nickases for cooperative genome engineering. Nat Biotechnol. 2013;31(9):833-8.

34. Chen B, Gilbert LA, Cimini BA, Schnitzbauer J, Zhang W, Li GW, Park J, Blackburn EH, Weissman JS, Qi LS, et al. Dynamic imaging of genomic loci in living human cells by an optimized CRISPR/Cas system. Cell. 2013;155(7):1479-91.

35. Fu Y, Foden JA, Khayter C, Maeder ML, Reyon D, Joung JK, Sander JD. High-frequency off-target mutagenesis induced by CRISPR-Cas nucleases in human cells. Nat Biotechnol. 2013;31(9):822-6.

36. Ran FA, Hsu PD, Lin CY, Gootenberg JS, Konermann S, Trevino AE, Scott DA, Inoue A, Matoba S, Zhang Y, et al. Double nicking by RNAguided CRISPR Cas9 for enhanced genome editing specificity. Cell. 2013;154(6):1380-9.

37. Cradick TJ, Fine EJ, Antico CJ, Bao G. CRISPR/Cas9 systems targeting betaglobin and CCR5 genes have substantial off-target activity. Nucleic Acids Res. 2013;41(20):9584-92.

38. Blitz IL, Biesinger J, Xie X, Cho KW. Biallelic genome modification in F Xenopus tropicalis embryos using the CRISPR/Cas system. Genesis. 2013;51(12):827-34 
39. Yang $H$, Wang $H$, Shivalila CS, Cheng AW, Shi L, Jaenisch R. One-step generation of mice carrying reporter and conditional alleles by CRISPR/ Cas-mediated genome engineering. Cell. 2013;154(6):1370-9.

40. Shi Z, Wang F, Cui Y, Liu Z, Guo X, Zhang Y, Deng Y, Zhao H, Chen Y. Heritable CRISPR/Cas9-mediated targeted integration in Xenopus tropicalis. FASEB J Off Publ Feder Am Soc Exp Biol. 2015;29(12):4914-23.

41. Wen L, Fu L, Guo X, Chen Y, Shi YB. Histone methyltransferase Dot1L plays a role in postembryonic development in Xenopus tropicalis. FASEB J Off Publ Feder Am Soc Exp Biol. 2015;29(2):385-93.

42. Pingault V, Ente D, Dastot-Le Moal F, Goossens M, Marlin S, Bondurand N. Review and update of mutations causing Waardenburg syndrome. Hum Mutat. 2010;31(4):391-406.

43. Guyonneau L, Murisier F, Rossier A, Moulin A, Beermann F. Melanocytes and pigmentation are affected in dopachrome tautomerase knockout mice. Mol Cell Biol. 2004;24(8):3396-403.

44. Nakajima K, Nakajima T, Takase M, Yaoita Y. Generation of albino Xenopus tropicalis using zinc-finger nucleases. Dev Growth Differ. 2012:54(9):777-84.

45. Wat MJ, Shchelochkov OA, Holder AM, Breman AM, Dagli A, Bacino C, Scaglia F, Zori RT, Cheung SW, Scott DA, et al. Chromosome 8p23.1 deletions as a cause of complex congenital heart defects and diaphragmatic hernia. Am J Med Genet A. 2009;149A(8):1661-77.

46. Akbas E, Senli H, Hallioglu O, Batmaz S, Erdogan NE. Association of pericentric inversion of chromosome 9 (inv[9][p11q13]) and genetic diseases: case report. Labmedicine. 2010;41(2):96-8.

47. Jiang W, Bikard D, Cox D, Zhang F, Marraffini LA. RNA-guided editing of bacterial genomes using CRISPR-Cas systems. Nat Biotechnol. 2013;31(3):233-9.

48. Lei Y, Guo X, Deng Y, Chen Y, Zhao H. Generation of gene disruptions by transcription activator-like effector nucleases (TALENs) in Xenopus tropicalis embryos. Cell Biosci. 2013;3(1):21.
49. Milet C, Maczkowiak F, Roche DD, Monsoro-Burq AH. Pax3 and Zic1 drive induction and differentiation of multipotent, migratory, and functional neural crest in Xenopus embryos. Proc Natl Acad Sci USA. 2013;110(14):5528-33.

50. Shi J, Severson C, Yang J, Wedlich D, Klymkowsky MW. Snail2 controls mesodermal BMP/Wnt induction of neural crest. Development. 2011;138(15):3135-45

51. Aybar MJ, Nieto MA, Mayor R. Snail precedes slug in the genetic cascade required for the specification and migration of the Xenopus neural crest. Development. 2003;130(3):483-94

52. Pattanayak V, Lin S, Guilinger JP, Ma EB, Doudna JA, Liu DR. High-throughput profiling of off-target DNA cleavage reveals RNA-programmed Cas9 nuclease specificity. Nature Biotechnol. 2013;31(9):839.

53. Fu YF, Foden JA, Khayter C, Maeder ML, Reyon D, Joung JK, Sander JD. High-frequency off-target mutagenesis induced by CRISPR-Cas nucleases in human cells. Nature Biotechnol. 2013;31(9):822.

54. Guilinger JP, Thompson DB, Liu DR. Fusion of catalytically inactive Cas9 to Fokl nuclease improves the specificity of genome modification. Nat Biotechnol. 2014;32(6):577-82.

55. Hwang WY, Fu Y, Reyon D, Maeder ML, Tsai SQ, Sander JD, Peterson RT, Yeh JR, Joung JK. Efficient genome editing in zebrafish using a CRISPRCas system. Nat Biotechnol. 2013;31(3):227-9.

56. Kim HJ, Lee HJ, Kim H, Cho SW, Kim JS. Targeted genome editing in human cells with zinc finger nucleases constructed via modular assembly. Genome Res. 2009;19(7):1279-88.

\section{Submit your next manuscript to BioMed Central and we will help you at every step:}

- We accept pre-submission inquiries

- Our selector tool helps you to find the most relevant journal

- We provide round the clock customer support

- Convenient online submission

- Thorough peer review

- Inclusion in PubMed and all major indexing services

- Maximum visibility for your research

Submit your manuscript at www.biomedcentral.com/submit

() Biomed Central 